\title{
Putting gene essentiality into context
}

One of the most fundamental pursuits of genetics has been to characterize genes that are essential for cellular or organismal viability. Such sets of genes - which are deemed the most important for survival - have numerous applications, including to design and engineer streamlined organisms with minimal genomes or to identify drug targets to treat infectious diseases.

This issue of Nature Reviews Genetics contains two articles that discuss gene essentiality from complementary angles. In their Review article, Rancati, Moffat, Typas and Pavelka ${ }^{1}$ focus on essentiality at the cellular level, leveraging their expertise across different prokaryotic and eukaryotic systems to describe how experimental tools and conceptual frameworks for gene essentiality have evolved and matured over time. Experimental identification and characterization of essential genes must overcome the hurdle that a classic knockout of an essential gene is inviable, so the authors discuss a timeline of progress in genetic techniques, from classic methods such as temperature-sensitive alleles to modern genome-wide CRISPR screens to infer the loss of lethal knockouts from a cell population. Considering essential gene sets across a range of organisms and contexts, the authors describe emerging overarching principles, such as the cellular functions and network neighbourhoods of essential genes and their relationships to genome size.

A major theme of the Review by Rancati et al. is that gene essentiality is highly context dependent, and they outline the various scenarios by which genetic and environmental contexts can alter gene essentiality. This context dependence has important implications, for example, when prioritizing therapeutic drug targets. For targeting a human pathogen, an ideal drug target will be essential to the pathogen in all the host microenvironments it encounters. By contrast, an emerging strategy for cancer therapy is synthetic lethality, whereby collateral toxicity to normal cells is minimized because a drug target is chosen that is essential only in cells with a known cancer driver mutation. Additionally, in both cases, the essentiality of an ideal drug target would need to have low evolvability. Such a property would minimize the opportunities for the emergence of antimicrobial or anticancer drug resistance due to adaptive selection of alternative genetic pathways.
In their Analysis article, Bartha, di Iulio, Venter and Telenti ${ }^{2}$ focus on essentiality in humans at the wholeorganism scale. In the absence of genetic manipulation tools in human populations, the authors describe and compare various bioinformatic tools that mine human population DNA sequence data to identify those genes that are the most intolerant to loss of function. Hence, essentiality is inferred through a high degree of evolutionary constraint across many human generations.

Similarly to Rancati et al., Bartha et al. describe the functions of the inferred essential genes and compare the gene sets across systems, here comparing the human population data with in vivo mouse knockout data and CRISPR knockout screens in human cells in vitro. Numerous important differences in the gene sets are described by the authors. Some differences arise because a cellular essential gene is only needed for that particular cell type in the culture conditions tested, whereas an organismal essential gene may be important in any cell type at any developmental stage. Bartha et al. also discuss technical reasons for differences, such as the heterozygous versus homozygous loss of function interrogated in the different systems. Beyond these differences, Bartha et al. discuss a core set of essential genes that are shared across these human and mouse data sets. These shared essential genes include several intriguing genes for which almost nothing is known about their functions, despite their near-ubiquitous importance.

In additional sections, Bartha et al. discuss the relationships between essential genes and diseaseassociated genes, as well as a framework for how customized essentiality metrics could extend beyond genes to also characterize non-coding elements.

Both articles emphasize that essentiality is more realistically scored as a quantitative measure than by a simplistic essential versus non-essential binary classification. This view is illustrated by the degree of essentiality across environmental and genetic contexts in Rancati et al. and in the numerical scores of intolerance to loss of function in Bartha et al. These articles thus highlight that any attempt to identify a definitive set of essential genes is almost certainly impossible. Instead, studies of essentiality are more enlightening when framed in terms of the degree of essentiality and the contexts in which different essentialities occur.

Rancati, G. et al. Nat. Rev. Genet. 19, 34-49 (2018).

Bartha, I. et al. Nat. Rev. Genet. 19, 51-62 (2018). 